\title{
Effect of serum inflammatory markers on the prognosis of adult and pediatric patients with Guillain-Barré syndrome
}

This article was published in the following Dove Press journal:

Neuropsychiatric Disease and Treatment

\author{
Ozlem Ethemoglu' \\ Mustafa Calik ${ }^{2}$ \\ 'Department of Neurology, Harran \\ University School of Medicine, \\ Șanlıurfa, Turkey; ${ }^{2}$ Department \\ of Pediatric Neurology, Harran \\ University School of Medicine, \\ Șanlıurfa, Turkey
}

Objective: The aim of this study was to evaluate blood neutrophil-lymphocyte ratio (NLR), platelet-lymphocyte ratio (PLR), C-reactive protein (CRP), and albumin levels for their prognostic value in adult Guillain-Barré syndrome (GBS-A) and pediatric Guillain-Barré syndrome (GBS-P) patients.

Patients and methods: We retrospectively analyzed the medical records of 68 GuillainBarré syndrome (GBS) patients (36 adults, 32 children) who were treated as inpatients at Harran University Faculty of Medicine, Neurology and Pediatric Neurology Departments. The pretreatment NLR, PLR, CRP, and albumin levels and Hughes scores at hospital admission, discharge, and third-month control were documented.

Results: In GBS-A patients, the mean CRP and NLR levels at admission/discharge and thirdmonth control were significantly higher, and the mean albumin level was significantly lower in the Hughes disability scale (HDS) $\geq 3$ group. In GBS-P group, the mean NLR level at third month was significantly higher in the HDS $\geq 3$ group. GBS-A patients had higher mean NLR, PLR, and CRP levels and lower mean albumin values than GBS-P patients. Both GBS-A and GBS-P patients had higher mean NLR, PLR, and CRP levels and lower mean albumin values than healthy controls. Only the albumin level of the GBS-A group was found to be a significant predictor of prognosis at discharge from hospital.

Conclusion: NLR, CRP, and albumin levels in the GBS-A group and NLR levels in the GBS-P group may be helpful in predicting the prognosis of the disease. The albumin level of GBS-A patients is an independent risk factor for prognosis at discharge from hospital.

Keywords: Guillain-Barré syndrome, albumin, neutrophil-lymphocyte ratio, platelet-lymphocyte ratio, $\mathrm{C}$-reactive protein

\section{Introduction}

Guillain-Barré syndrome (GBS), an acute immune-mediated disorder of peripheral nerves and spinal roots, is the most common peripheral nervous system disorder. Immune response is the major cause of GBS. In this syndrome, autoantibodies triggered by previous infections cross-react with gangliosides and result in peripheral nervous system injury. ${ }^{1}$ Moreover, it has been recently shown that inflammatory factors apart from cellular and humoral immunity play a role in the GBS pathogenesis. ${ }^{2}$ Recently, neutrophil-lymphocyte ratio (NLR) is known to be widely used as a marker of inflammation and a predictor of prognosis in various disorders. Higher NLR values were shown to be an independent predictor of a worse prognosis among patients with acute ischemic stroke, cardiac disorders, and cancer. ${ }^{3-5}$ Likewise, increased
Correspondence: Ozlem Ethemoglu Department of Neurology, Harran University School of Medicine,

TR-63 I00, Șanlıurfa, Turkey

Tel +904143444444

$\mathrm{Fax}+904143183192$

Email ozlem_uzunkaya@hotmail.com 
platelet-lymphocyte ratio (PLR) was shown to predict a poor prognosis in patients with acute stroke and malignancy. 5,6

C-reactive protein (CRP) is an acute-phase reactant whose level is elevated by infectious, inflammatory, autoinflammatory, and autoimmune disorders. ${ }^{7.8}$ Increased CRP levels have been associated with poor prognosis among patients with subarachnoid hemorrhage. ${ }^{9}$ Furthermore, a recent study has indicated its role for the determination of the prognosis of functional disability in stroke patients. ${ }^{10}$ Albumin is a negative acute-phase protein affected by inflammation. ${ }^{11}$ A recent study of adult GBS (GBS-A) patients demonstrated that hypoalbuminemia was negatively correlated with disability at hospital admission and discharge. ${ }^{12}$

To the best of our knowledge, our study is the first one that quantifies serum NLR, PLR, CRP, and albumin levels and correlates these markers with prognosis among GBS-A and pediatric GBS (GBS-P) patients. Our primary objective was to investigate the correlation of serum inflammatory markers with short- and long-term prognosis of GBS patients.

\section{Patients and methods}

We retrospectively analyzed the medical records of a total of 68 GBS patients (36 adults, 32 children) who were treated as inpatients at Harran University Faculty of Medicine, Neurology and Pediatric Neurology Departments, between January 2013 and July 2017 and followed clinically thereafter. The control group consisted of 33 healthy adults and 30 healthy children. The exclusion criteria included local or systemic infections, steroid use, malignancy, and chronic diseases such as hematological, autoimmune, renal, cardiovascular, and hepatic disorders. Our study was designed according to the Declaration of Helsinki and approved by the local ethics committee of Harran University Medical Faculty (HUMF). Patients' consent to review their medical records was exempted by the local ethics committee of HUMF as the study involved the collection of existing data and was recorded taking care of the confidentiality of the data.

Admission and outpatient follow-up records of all patients were retrospectively reviewed. Age, sex, and the results of neurological examination and electrophysiological studies were recorded.

Complete blood count, renal function test, and hepatic function test were performed within 24 hours of hospital admission and before the treatment, and serum CRP, albumin, neutrophil, lymphocyte, thrombocyte, CRP, NLR, and PLR levels were recorded. All patients underwent electromyography in the first 2 days of hospitalization. The disorder was grouped into acute inflammatory demyelinating polyradiculoneuropathy
(AIDP), acute motor axonal neuropathy (AMAN), and acute motor sensory axonal neuropathy (AMSAN) subgroups on the basis of electrophysiological findings. The patients were categorized into the demyelinating form (AIDP) and axonal form (AMAN and AMSAN) subtypes.

To determine the neurological disability, Hughes disability scale (HDS) was determined at admission, discharge, and 3 months later. According to HDS, stage 1-3 was considered to represent good prognosis, and stage 3 or higher was considered to represent worse prognosis.

\section{HDS}

In HDS, the patients were scored between 0 and 6 points. Accordingly, grade $0=$ healthy; grade $1=$ minor signs and symptoms; grade $2=$ being able to walk without support $5 \mathrm{~m}$ across but being unable to run; grade $3=$ being able to walk with support or an appliance such as walker; grade $4=$ being confined to bed or chair bound; grade $5=$ requiring assisted ventilation; and grade $6=$ death. ${ }^{13} \mathrm{~A}$ disability score of 3 or greater was considered to predict poor prognosis, while a score of 2 or less was considered to predict good prognosis.

\section{Laboratory studies}

Venous blood samples were drawn from all patients within the first 24 hours after admission. NLR was calculated by dividing the neutrophil count by lymphocyte count, and PLR by dividing the thrombocyte count by lymphocyte count. NLR, PLR, CRP, albumin, neutrophil, lymphocyte, and thrombocyte levels were recorded.

\section{Statistical analyses}

All statistical analyses were performed using the Statistical Package for Social Sciences for Windows version 22.0 (IBM Corporation, Armonk, NY, USA) software package. Descriptive statistics included mean $\pm \mathrm{SD}$, number, and percentage. Student's $t$-test was used to compare normally distributed continuous variables, while Mann-Whitney $U$-test was used to compare non-normally distributed variables. Categorical variables were compared using chi-square and Fisher's exact tests. Logistic regression analysis was performed to determine risk factors of short- and long-term prognosis. A $p$-value of $<0.05$ was considered statistically significant for all comparisons and correlations.

\section{Results}

This study included a total of 36 adult (mean age $48.5 \pm$ 18.95 years) patients, 32 pediatric (mean age $5.68 \pm 4.69$ years) patients, 33 healthy adults (mean age $41.69 \pm 20.35$ years), and 
Table I Comparisons of inflammatory markers between AIDP and axonal subgroups

\begin{tabular}{|c|c|c|c|c|c|c|}
\hline & \multicolumn{2}{|l|}{ Adult } & \multicolumn{2}{|l|}{ Pediatric } & \multirow[t]{2}{*}{$p$} & \multirow[t]{2}{*}{ pl } \\
\hline & AIDP $(n=22)$ & Axonal $(n=14)$ & AIDP $(n=18)$ & Axonal $(n=14)$ & & \\
\hline Age, years & $50.90 \pm 20.94$ & $44.7 \mathrm{I} \pm 15.27$ & $6.55 \pm 4.35$ & $4.56 \pm 5.04$ & 0.346 & 0.059 \\
\hline NLR & $3.44 \pm 1.94$ & $3.98 \pm 1.34$ & $1.43 \pm 0.83$ & $2 .|8 \pm 1.3|$ & 0.368 & 0.070 \\
\hline PLR & $162.58 \pm 62.18$ & $|7| .75 \pm 69.1 \mid$ & $104.19 \pm 35.85$ & II $8.37 \pm 72.67$ & 0.682 & 0.985 \\
\hline CRP, mg/dL & $0.79 \pm 1.88$ & $0.82 \pm 0.47$ & $0.05 \pm 0.07$ & $0.20 \pm 0.31$ & 0.950 & 0.122 \\
\hline Albumin, gr/dL & $3.68 \pm 0.59$ & $3.3 I \pm 0.64$ & $4.29 \pm 0.39$ & $3.98 \pm 0.47$ & 0.121 & 0.060 \\
\hline
\end{tabular}

Notes: The data were presented as mean \pm SD. p: significance between AIDP and axonal group in GBS-A patients, pl: significance between AIDP and axonal group in GBS-P patients.

Abbreviations: AIDP, acute inflammatory demyelinating polyradiculoneuropathy; CRP, C-reactive protein; NLR, neutrophil-lymphocyte ratio; PLR, platelet-lymphocyte ratio.

30 healthy children (mean age $6.45 \pm 4.32$ years). As the thirdmonth follow-up data of seven patients in the GBS-A group and four patients in the GBS-P group could not be accessed, these patients were evaluated in the hospital admission and discharge groups only. A total of 29 patients in the GBS-P group were administered intravenous immunoglobulin; two were administered plasmapheresis; and one patient was administered intravenous immunoglobulin + plasmapheresis. A total of 22 (61.1\%) GBS-A patients had AIDP; seven (19.4\%) had AMAN, and seven (19.4\%) had AMSAN. A total of 18 (56.3\%) GBS-P patients had AIDP; 13 (40.6\%) had AMAN; and one (3.1\%) had AMSAN. No significant relationship was found between NLR, PLR, CRP, and albumin levels and the demyelinating and axonal subtypes in both the GBS-A and GBS-P patient groups (Table 1).

In GBS-A patients, the mean CRP and NLR levels at admission/discharge and third-month control were significantly higher, and the mean albumin level was significantly lower in the HDS $\geq 3$ group. In the GBS-P group, on the other hand, the mean NLR level at third month was significantly higher in the HDS $\geq 3$ group, but there was no significant difference with the other inflammatory markers. In the pediatric group, the mean age of the HDS $\geq 3$ group was significantly lower than that of the HDS $<3$ group (Table 2).

Comparison of the GBS-A and GBS-P groups with respect to inflammatory parameters revealed that the former group had a significantly higher mean CRP, NLR, and PLR levels and a significantly lower mean albumin level (Table 3).

Comparison of the mean levels of the inflammatory markers in both the GBS-A and GBS-P groups with those of the control group showed that both patient groups had significantly higher levels of CRP, NLR, and PLR levels and a significantly lower level of albumin compared to the controls (Table 4).

The number of patients in the HDS $\geq 3$ group at thirdmonth control was significantly greater in the GBS-A group compared to the GBS-P group ( $p=0.032$ ).

According to logistic regression analysis, only the albumin level of the GBS-A group was found to be a significant predictor of prognosis at discharge from hospital $(p=0.040)$.

Table 2 The demographic and laboratory characteristics of GBS-A and GBS-P patients

\begin{tabular}{|c|c|c|c|c|c|c|c|c|c|}
\hline & \multicolumn{2}{|l|}{ Admission } & \multicolumn{2}{|l|}{ Discharged } & \multicolumn{2}{|l|}{ Control } & \multirow[t]{2}{*}{$p$} & \multirow[t]{2}{*}{ pl } & \multirow[t]{2}{*}{$p^{2}$} \\
\hline & HDS-A $<3$ & HDS-A $\geq 3$ & HDS-D $<3$ & HDS-D $\geq 3$ & HDS-C $<3$ & HDS-C $\geq 3$ & & & \\
\hline GBS-A & $n=9$ & $n=27$ & $n=18$ & $n=18$ & $n=13$ & $n=15$ & & & \\
\hline Age, years & $43.66 \pm 19.66$ & $50.11 \pm 18.80$ & $44.66 \pm 18.67$ & $52.33 \pm 18.96$ & $45.46 \pm 19.70$ & $47.33 \pm 13.98$ & 0.405 & 0.230 & 0.783 \\
\hline NLR & $2.29 \pm 0.88$ & $4.10 \pm 1.72$ & $2.92 \pm 1.69$ & $4.38 \pm 1.49$ & $2.80 \pm 1.29$ & $4.25 \pm 1.39$ & 0.000 & 0.009 & 0.010 \\
\hline PLR & $163.83 \pm 63.17$ & $166.92 \pm 65.63$ & $172.58 \pm 67.40$ & $|59.7| \pm 6 \mid .97$ & $|80.44 \pm 7| .06$ & $|56.40 \pm 52.2|$ & 0.902 & 0.555 & 0.513 \\
\hline CRP, mg/dL & $0.12 \pm 0.12$ & $1.03 \pm 1.66$ & $0.34 \pm 0.36$ & $1.27 \pm 1.99$ & $0.34 \pm 0.35$ & $0.76 \pm 0.46$ & 0.000 & 0.001 & 0.036 \\
\hline Albumin, gr/dL & $3.97 \pm 0.20$ & $3.45 \pm 0.65$ & $3.99 \pm 0.45$ & $3.15 \pm 0.49$ & $3.92 \pm 0.53$ & $3.17 \pm 0.52$ & 0.007 & 0.000 & 0.003 \\
\hline GBS-P & $n=6$ & $n=26$ & $n=I I$ & $\mathbf{n}=\mathbf{2} \mathbf{I}$ & $n=19$ & $n=7$ & p3 & $p 4$ & p5 \\
\hline Age, years & $6.93 \pm 4.0$ & $5.39 \pm 4.86$ & $6.86 \pm 4.23$ & $5.06 \pm 4.90$ & $6.75 \pm 4.72$ & $3.11 \pm 4.12$ & 0.308 & 0.096 & 0.030 \\
\hline NLR & $1.51 \pm 0.86$ & $1.82 \pm 1.17$ & I. $.47 \pm 0.86$ & $1.91 \pm 1.22$ & $1.39 \pm 0.86$ & $2.80 \pm 1.35$ & 0.482 & 0.250 & 0.004 \\
\hline PLR & $114.83 \pm 43.95$ & $110.70 \pm 57.53$ & $|02.2| \pm 34.86$ & $116.33 \pm 62.80$ & $95.09 \pm 34.70$ & $|45.63 \pm 8| .35$ & 0.359 & 0.766 & 0.066 \\
\hline CRP, mg/dL & $0.07 \pm 0.11$ & $0.13 \pm 0.24$ & $0.05 \pm 0.08$ & $0.15 \pm 0.26$ & $0.13 \pm 0.26$ & $0.10 \pm 0.15$ & 0.492 & 0.170 & 0.638 \\
\hline Albumin, gr/dL & $4.38 \pm 0.39$ & 4. $10 \pm 0.45$ & $4.35 \pm 0.30$ & $4.06 \pm 0.49$ & $4.27 \pm 0.4 \mathrm{I}$ & $3.91 \pm 0.52$ & 0.179 & 0.088 & 0.079 \\
\hline
\end{tabular}

Notes: $p$, significance between adult HDS-A $<3$ and adult HDS-A $\geq 3$; $p$ I, significance between adult HDS-D $<3$ and adult HDS-D $\geq 3$; $p 2$, significance between adult HDS$C<3$ and adult HDS-C $\geq 3 ; p 3$, significance between pediatric HDS-A $<3$ and pediatric HDS-A $\geq 3$; $p 4$, pediatric HDS-D $<3$ and pediatric HDS-D $\geq 3$; $p 5$, pediatric HDS-C $<3$ and pediatric HDS-C $\geq 3$.

Abbreviations: CRP, C-reactive protein; GBS, Guillain-Barré syndrome; GBS-A, adult GBS; GBS-P, pediatric GBS; HDS-A, Hughes disability scale-admission; HDS-C, Hughes disability scale - control; HDS-D, Hughes disability scale - discharged; NLR, neutrophil-lymphocyte ratio; PLR, platelet-lymphocyte ratio. 
Table 3 Comparisons of inflammatory markers between adults and children

\begin{tabular}{llll}
\hline & GBS-A & GBS-P & p-value \\
\hline NLR & $3.65 \pm I .73$ & $1.76 \pm 1.21$ & 0.000 \\
PLR & $166.15 \pm 64.14$ & $111.48 \pm 54.62$ & 0.000 \\
CRP, mg/dL & $0.80 \pm 1.48$ & $0.16 \pm 0.30$ & 0.000 \\
Albumin, gr/dL & $3.52 \pm 0.63$ & $4.15 \pm 0.44$ & 0.000 \\
\hline
\end{tabular}

Abbreviations: CRP, C-reactive protein; GBS, Guillain-Barré syndrome; GBS-A, adult GBS; GBS-P, pediatric GBS; NLR, neutrophil-lymphocyte ratio; PLR, plateletlymphocyte ratio.

\section{Discussion}

GBS is usually diagnosed on the basis of a patient's signs and symptoms with the assistance of laboratory cerebrospinal fluid findings and electrophysiological criteria. ${ }^{14}$ Among GBS patients, the role of some biomarkers such as myelin basic protein, neurofilaments, anti-ganglioside antibodies, neuron-specific enolase, hypocretin-1, tumor necrosis factor, chemokines, and complements in disease pathology and prognosis has been examined. ${ }^{15,16}$ There is a limited number of studies in the literature which have explored the correlation between GBS-A patients and serum inflammatory markers. To date, no study has assessed the association between GBS-P patients and serum NLR, PLR, CRP, and albumin levels.

Recent studies have indicated that NLR and PLR are novel markers that are able to predict the prognosis of various disorders. ${ }^{3-6}$ In a study by Ozdemir ${ }^{12}$ on GBS-A patients, there was no significant correlation between NLR and PLR and admission and discharge disability. In another study, Geyik et $\mathrm{al}^{17}$ reported that NLR level at hospital admission showed a significant negative correlation with the posttreatment HDS. In this study, we found higher NLR levels at admission, discharge, and third-month control in the GBS-A group, whereas it was higher only at the third-month control in the GBS-P group. Furthermore, increased NLR levels were significantly correlated with worse prognosis.

In addition to infectious and inflammatory conditions, autoinflammatory and autoimmune disorders also increase
CRP levels. ${ }^{8,9} \mathrm{CRP}$ is involved in innate immunity, increasing levels of inflammation when not accompanied by any viral or bacterial pathogen, and plays a role in the development of autoimmune disorders by binding to autoantigens. ${ }^{12,18}$ A literature search on PubMed revealed only one study investigating the relationship between GBS-A patients and CRP levels. In that study, Vaishnavi et al ${ }^{19}$ found higher CRP levels in GBS-A patients compared to the controls, whereas they reported no correlation between electrophysiological subgroups and CRP levels. In accordance with the literature, mean CRP levels were higher in both the GBS-A and GBS-P groups compared to the control group, but there was no significant difference between subgroups. The mean CRP level was correlated with poor prognosis among GBS-A patients.

Hypoalbuminemia is a negative acute-phase protein indicating inflammation. Various studies have indicated an association between hypoalbuminemia and other acutephase proteins and increased CRP level. ${ }^{20,21}$ It was previously reported to be correlated with worse prognosis among patients with ischemic stroke..$^{22} \mathrm{~A}$ study on rats observed an improvement in neurological deficit and a reduction in bloodbrain barrier when albumin treatment was administered following acute intracortical hematoma formation. ${ }^{23}$ Another study investigated the relationship between albumin level and disability at hospital admission and discharge in GBS. It revealed a negative correlation between albumin level and HDS at admission and discharge. ${ }^{12}$ Fokkink et a ${ }^{24}$ reported an increased rate of respiratory failure among GBS-A patients with hypoalbuminemia prior to treatment. The mean albumin level of the GBS-A group was significantly lower among the HDS $\geq 3$ group at admission, discharge, and control visit, whereas there was no significant relationship between albumin level and disability for the GBS-P group.

Former studies have failed to indicate any correlation between the prognosis of GBS-P patients and patients' age. However, we revealed that the mean age at third-month control was significantly lower in the HDS $\geq 3$ group.

Table 4 Comparisons of inflammatory markers between GBS-A and GBS-P patients with control groups

\begin{tabular}{lllllll}
\hline & GBS-A & GBS-AC & GBS-P & GBS-PC & P & PI \\
\hline Age, years & $48.50 \pm \mid 8.95$ & $42.45 \pm \mid 9.86$ & $5.68 \pm 4.69$ & $6.73 \pm 3.95$ & 0.200 & 0.312 \\
NLR & $3.65 \pm 1.73$ & $1.91 \pm 0.59$ & $1.79 \pm 1.12$ & $1.18 \pm 0.61$ & 0.000 & 0.041 \\
PLR & $166.15 \pm 64.14$ & $120.61 \pm 23.8 \mid$ & $110.39 \pm 54.50$ & $101.41 \pm \mid 2.92$ & 0.000 & 0.933 \\
CRP, mg/dL & $0.80 \pm 1.48$ & $0.12 \pm 0.12$ & $0.21 \pm 0.34$ & $0.05 \pm 0.08$ & 0.000 & 0.020 \\
Albumin, gr/dL & $3.52 \pm 0.63$ & $3.84 \pm 0.42$ & $4.15 \pm 0.44$ & $4.05 \pm 0.23$ & 0.026 & 0.242 \\
\hline
\end{tabular}

Notes: $p$, significance between GBS-A and GBS-AC; $p$ I, significance between GBS-P and GBS-PC.

Abbreviations: CRP, C-reactive protein; GBS, Guillain-Barré syndrome; GBS-A, adult GBS; AC, adult control; GBS-P, pediatric GBS; PC, pediatric control; NLR, neutrophillymphocyte ratio; PLR, platelet-lymphocyte ratio. 
Prior studies have reported that increased NLR and PLR levels are an independent risk factor for cardiovascular disease and an indicator of worse prognosis. ${ }^{25-27} \mathrm{~A}$ recent metaanalysis indicated that increased CRP level was significantly correlated with cardiovascular disease, ischemic stroke, and vascular and nonvascular death among people without a history of vascular disease. ${ }^{28}$ Moreover, it is an established fact that cardiovascular complications are one of the major causes of death among GBS patients in both acute stage and convalescence period. ${ }^{26}$ In the current study, the mean values of serum inflammatory markers were significantly higher in both pediatric and adult patients than those of controls. Various cardiovascular complications (arrhythmia, hypertension, and hypotension) were observed in eight (22.8\%) patients in the GBS-A group and three $(9.3 \%)$ patients in the GBS-P group. Nevertheless, none of our patients died from cardiovascular complications during the treatment period. Due to the small number of patients in our study group, the relationship between inflammatory parameters and patients with cardiac complications was not assessed. The relationship between cardiac complications and inflammatory parameters requires further investigation.

GBS-P patients enjoy a better prognosis than their adult counterparts. About $72 \%$ of GBS-P patients are able to walk unassisted at the end of the first year. That ratio is about two times of that of GBS-A patients..$^{29,30}$ In our study, the number of patients being able to walk without assistance at thirdmonth control was significantly greater in the GBS-P group compared to the GBS-A group. Significantly higher levels of the inflammatory markers such as CRP, NLR, and PLR but a lower level of albumin in GBS-A patients compared to GBS-P patients suggest an increased level of inflammation among adults. Hence, excessive inflammation in the adult patient group may be the cause of a greater disability rate in this group compared to the children. The limitations of our study included a small sample size and a short functional follow-up.

\section{Conclusion}

We believe that our study is valuable, because no other study has yet determined the effect of serum inflammatory markers on the prognosis of GBS-A and GBS-P patients. GBS patients have a variable clinical course and prognosis. Prediction of patients who would have a grave prognosis at an early stage is highly relevant for the management of these patients. Our study indicates that inflammatory markers including serum NLR, PLR, CRP, and albumin levels may be used as cheaper, more readily available, and more rapidly studied markers for the prediction of the prognosis of GBS. Regression analysis showed that the albumin level of the GBS-A group was an independent prognostic factor at discharge from hospital. The GBS-P group had a better prognosis than the GBS-A group at third-month control. However, prospective studies with larger volume and longer follow-up are needed to assess the reliability of these markers for making the diagnosis of GBS, monitoring patients, and determining their prognosis.

\section{Disclosure}

The authors report no conflicts of interest in this work.

\section{References}

1. Van den Berg B, Walgaard C, Drenthen J, Fokke C, Jacobs BC, vanDoorn PA. Guillain-Barré syndrome: pathogenesis, diagnosis, treatment and prognosis. Nat Rev Neurol. 2014;10(8):469-482.

2. Lu MO, Zhu J. The role of cytokines in Guillain-Barré syndrome. J Neurol. 2011;258(4):533-548.

3. Qun S, Tang Y, Sun J, et al. Neutrophil-to-lymphocyte ratio predicts 3-month outcome of acute ischemic stroke. Neurotox Res. 2017;31(3): 444-452.

4. Imtiaz F, Shafique K, Mirza SS, Ayoob Z, Vart P, Rao S. Neutrophil lymphocyte ratio as a measure of systemic inflammation in prevalent chronic diseases in Asian population. Int Arch Med. 2012;5(1):2.

5. Auezova R, Ryskeldiev N, Doskaliyev A, et al. Association of preoperative levels of selected blood inflammatory markers with prognosis in gliomas. Onco Targets Ther. 2016;11(9):6111-6117.

6. Altintas O, Altintas MO, Tasal A, Kucukdagli OT, Asil T. The relationship of platelet-to-lymphocyte ratio with clinical outcome and final infarct core in acute ischemic stroke patients who have undergone endovascular therapy. Neurol Res. 2016;38(9):759-765.

7. Du Clos TW. C-reactive protein as a regulator of autoimmunity and inflammation. Arthritis Rheum. 2003;48(6):1475-1477.

8. Szalai AJ. C-reactive protein and autoimmune disease: Facts and conjectures. Clin Dev Immunol. 2004;11:221-226.

9. Romero FR, Bertolini EF, Figueiredo EG, Teixeira MJ. Serum C-reactive protein levels predict neurological outcome after aneurysmal subarachnoid hemorrhage. Arq Neuropsiquiatr. 2012;70:202-205.

10. Song IU, Kim YD, Kim JS, Lee KS, Chung SW. Can high sensitivity C-reactive protein and plasma homocysteine levels independently predict the prognosis of patients with functional disability after first ever ischemic stroke? Eur Neurol. 2010;64:304-310.

11. Don BR, Kaysen G. Serum albumin: relationship to inflammation and nutrition. Semin Dial. 2004;17(6):432-437.

12. Ozdemir HH. Analysis of the albumin level, neutrophil-lymphocyte ratio, and platelet-lymphocyte ratio in Guillain-Barré syndrome. Arq Neuropsiquiatr. 2016;74(9):718-722.

13. Hughes RA, Newsom-Davis JM, Perkin GD, Pierce J. Controlled trial prednisolone in acute polyneuropathy. Lancet. 1978;2:750-753.

14. Hughes RAC, Cornblath DR. Guillain-Barré Syndrome. Lancet. 2005; 366:1653-1656.

15. Wang Y, Sun S, Zhu J, Cui L, Zhang HL. Biomarkers of Guillain-Barré syndrome: some recent progress, more still to be explored. Mediators Inflamm. 2015;2015:564098.

16. Petzold A, Hinds N, Murray NM, et al. CSF neurofilament levels: a potential prognostic marker in Guillain-Barré syndrome. Neurology. 2006; 67(6):1071-1073.

17. Geyik S, Bozkurt H, Neyal M, Yigiter R, Kuzudisli S, Kul S. The clinical significance of the neutrophil-to-lymphocyte ratio in patients with Guillain-Barré syndrome independent of infection. Med Sci Discov. 2016;3(8):305-311. 
18. Peaceman AM, Andrews WW, Thorp JM, et al. Fetal fibronectin as a predictor of preterm birth in patients with symptoms: A multicenter trial. Am J Obstet Gynecol. 1997;177:13-18.

19. Vaishnavi C, Kapoor P, Behura C, Singh SK, Prabhakar S. C-reactive protein in patients with Guillain Barré syndrome. Indian J Pathol Microbiol. 2014;57(1):51-54.

20. Bologa RM, Levine DM, Parker TS, et al. Interleukin-6 predicts hypoalbuminemia, hypocholesterolemia, and mortality in hemodialysis patients. Am J Kidney Dis. 1998;32:107-114.

21. Yeun JY, Levine RA, Mantadilok V, Kaysen GA. C-Reactive protein predicts all-cause and cardiovascular mortality in hemodialysis patients. Am J Kidney Dis. 2000;35:469-476.

22. Cho YM, Choi IS, Bian RX, Kim JH, Ham JY, Lee SG. Serum albumin at admission for prediction of functional outcome in ischemic stroke patients. Neurol Sci. 2008;29:445-449.

23. Belayev L, Saul I, Busto R, et al. Albumin treatment reduces neurological deficit and protects blood-brain barrier integrity after acute intracortical hematoma in the rat. Stroke. 2005;36(2):326-331.

24. Fokkink WR, Walgaard C, Kuitwaard K, Tio-Gillen AP, van Doorn PA, Jacobs BC. Association of albumin levels with outcome in intravenous immunoglobulin-treated Guillain-Barré syndrome. JAMA Neurol. 2017;74(2):189-196.
25. Demirkol S, Balta S, Unlu M, et al. Neutrophils/lymphocytes ratio in patients with cardiac syndrome $\mathrm{X}$ and its association with carotid intima media thickness. Clin Appl Thromb Hemost. 2014;20(3):250-255.

26. Van den Berg B, Bunschoten C, Van Doorn PA, Jacobs BC. Mortality in Guillain-Barré syndrome. Neurology. 2013;80(18):1650-1654.

27. Li H, Zhou Y, Ma Y, Han S, Zhou L. The prognostic value of the platelet-to-lymphocyte ratio in acute coronary syndrome: a systematic review and meta-analysis. Kardiol Pol. 2017;75(7):666-673.

28. Emerging Risk Factors Collaboration, Kaptoge S, Kaptoge S, et al. C-reactive protein concentration and risk of coronary heart disease, stroke, and mortality: an individual participant meta-analysis. Lancet. 2010; 375(9709):132-140.

29. Korinthenberg R, Schessl J, Kirschner J. Clinical presentation and course of childhood Guillain-Barré syndrome: a prospective multicentre study. Neuropediatrics. 2007;38:10-17.

30. Sarada C, Tharakan JK, Nair M. Guillain-Barre syndrome: A prospective clinical study in 25 children and comparison with adults. Ann Trop Paediatr. 1994;14:281-286.

\section{Publish your work in this journal}

Neuropsychiatric Disease and Treatment is an international, peerreviewed journal of clinical therapeutics and pharmacology focusing on concise rapid reporting of clinical or pre-clinical studies on a range of neuropsychiatric and neurological disorders. This journal is indexed on PubMed Central, the 'PsycINFO' database and CAS, and is the official journal of The International Neuropsychiatric Association (INA). The manuscript management system is completely online and includes a very quick and fair peer-review system, which is all easy to use. Visit http://www.dovepress.com/testimonials.php to read real quotes from published authors. 